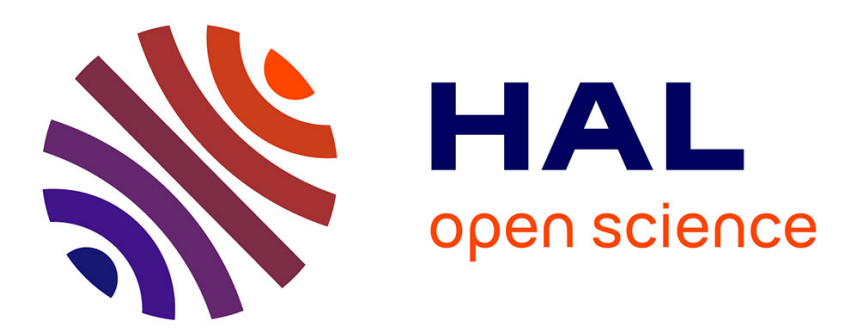

\title{
Narrow electromagnetic beam transmission associated to the Dirac cones in a graphenelike photonic crystal Kang Wang
}

\section{To cite this version:}

Kang Wang. Narrow electromagnetic beam transmission associated to the Dirac cones in a graphenelike photonic crystal. Physical Review B, 2021, 103, pp.235107. 10.1103/PhysRevB.103.235107 . hal-03087584v3

\author{
HAL Id: hal-03087584 \\ https://hal.science/hal-03087584v3
}

Submitted on 28 Jul 2021

HAL is a multi-disciplinary open access archive for the deposit and dissemination of scientific research documents, whether they are published or not. The documents may come from teaching and research institutions in France or abroad, or from public or private research centers.
L'archive ouverte pluridisciplinaire HAL, est destinée au dépôt et à la diffusion de documents scientifiques de niveau recherche, publiés ou non, émanant des établissements d'enseignement et de recherche français ou étrangers, des laboratoires publics ou privés. 


\title{
Narrow electromagnetic beam transmission associated to the Dirac cones in a graphenelike photonic crystal
}

\author{
Kang Wang (1) \\ Laboratoire de Physique des Solides, CNRS, Université Paris-Saclay, 91405 Orsay, France
}

(Received 22 January 2021; revised 6 May 2021; accepted 24 May 2021; published 1 June 2021)

\begin{abstract}
We study the transmission of narrow electromagnetic beams associated to different $K$ valleys, from both the armchair and zigzag edges, in a graphenelike triangular metallic photonic crystal. Due to the metallic nature of the structure, the propagation of an electromagnetic wave, with the wave vector close to the quasitriangular isofrequency contours of the Dirac cones resulting from the band trigonal warping, can be considered as being based on a series of bonding or antibonding states, formed by the local resonance modes in the unit cells. The transmission of a narrow incident beam, of the width of the crystal period size, depends on the local structure configurations in the beam impact zone that determine the distribution of the excited local resonance modes as well as the coupling between the latter and the incident field, thus the beam propagation in different directions. This investigation allows us to understand the interaction between an incident field and the resonance states associated to the Dirac cones at elementary structure scales reaching the size of these states. It can find application in valley photonics for transport tuning and optimization.
\end{abstract}

DOI: 10.1103/PhysRevB.103.235107

\section{INTRODUCTION}

Graphenelike photonic crystals are widely studied both for their fundamental interest and application potential. Indeed, these structures reproduce the electronic bands of the atomic structures by creating Dirac cones in their frequency bands [1]. An electromagnetic (EM) emission can be coupled to such structures and the resulting spectrum modifications provide information on the dispersion properties of the Dirac cones as well as their coupling with the incident field. Being geometrically and materially tunable, such photonic crystals enable investigations of the physical properties inaccessible in atomic structures by allowing structure configurations and modulations unrealizable in the latter. Many fundamental phenomena, specific to the Dirac cones, including conical diffraction, gap soliton, pseudodiffusive transmission, quantum Hall-like effect, zero refractive index, and valley-polarized beam propagation [1-5] have been investigated. Potential applications, such as valley photonics exploring optically the valley degree of freedom to control and manipulate the light beam propagation, analogous to valleytronics [6], have recently been proposed for information processing [7]. Indeed, it is shown that, by lifting the inversion symmetry in the structures, it is possible to achieve photonic topological insulators for largescale photonic integrations [8] and independent control of valley-dependent transportation [7].

Dirac cones can emerge in various dielectric [1-4,9] and metallic [10-12] photonic crystals. In the former, the band formation can be described by a nearly-free-photon approximation [13], while, in the latter, the low-frequency EM bands are formed by cavitylike local resonance modes con-

*kang.wang@universite-paris-saclay.fr fined inside the structure tiles delimited by metallic elements $[11,12,14]$. Such local resonance modes follow a honeycomb distribution in a triangular metallic structure, which is thus equivalent to a honeycomb atomic one.

It is known that, due to the local threefold symmetry in a honeycomb structure, the Dirac cones undergo trigonal warping for frequencies departing from the Dirac level [15], lifting the degeneracy between two inequivalent cones. The group velocity distribution becomes increasingly anisotropic. When the isofrequency contours of the Dirac cones become nearly triangular, the wave propagation will follow the $\Gamma-K$ directions that are perpendicular to the isofrequency contours, and a beam propagating in such a structure undergoes splitting and self-collimation associated to different $K$ valleys [5]. This provides an alternative way for wave propagation control and beam manipulation. Studies on $K$-valley dependent EM beam propagation, associated to the lower Dirac cones in dielectric photonic crystals $[5,16]$ and to the upper cones in analogous situations of electron beams in atomic graphenes [17,18], are both carried out. Beam splitting and self-collimation are obtained for the zigzag and armchair edge incidences.

Due to the huge value of the dielectric function of the metallic elements, metallic photonic crystals need only a few number of periods to achieve band gaps [19], allowing smaller sample sizes in practice. More particularly, in a metallic photonic crystal, the eigenmodes of the low frequency crystal wave function can be constructed by the combination of the local resonance modes mentioned above in a tight-binding (TB) model $[11,12,14]$. An EM wave propagating in a metallic photonic crystal will then follow the structural tile distribution, through the coupling between the local resonance modes. We have shown previously that an EM beam propagation associated to trigonally warped Dirac cones in a graphenelike triangular metallic photonic crystal display drastically 
different behaviors as compared to the dielectric and other analogous systems, due to the EM wave function symmetry along the $\Gamma-K$ lines and the metallic nature of the structure. Namely, at the armchair edge, the incident beam associated to the lower Dirac cone is collimated to one central beam, and that associated to the upper cone is split to two beams with a $2 \pi / 3$ angle. The other expected beams are all strongly inhibited [20].

In this work we report a study of the transmission of narrow incident beams, of the widths of the crystal period scales, from both the armchair and zigzag edges in a triangular metallic photonic crystal, associated to the trigonally warped Dirac cones. We discuss the EM frequency band structure close to the quasitriangular isofrequency contours of a Dirac cone and show that the beam transmission depends on the position and orientation of unit cells along the propagation directions in the beam impact zone that will determine the characteristics of the excited local resonance modes and the coupling between the latter and the incident field, thus the beam propagation in different directions.

\section{BAND STRUCTURE CLOSE TO QUASITRIANGULAR ISOFREQUENCY CONTOURS OF A DIRAC CONE}

A triangular lattice [Fig. 1(a)] belongs to the symmetry group $D_{6 h}$ and contains two families of triangular tiles, pointing, respectively, up- and downward in the figure. These two families of tiles will be referred to as $A$ and $B$ tiles hereinafter. The metallic photonic crystal is constructed by infinitely long cylinders, with radius $r=0.25 a$ ( $a$ being the lattice constant) and dielectric constant set to negative infinity, placed at the lattice nodes in an air background. The EM frequency band diagram for TM polarization (the electric field parallel to the $z$ axis), obtained in the previous work [20] by solving Maxwell's equations using the finite-difference time-domain (FDTD) method [11], is reproduced in Fig. 1(b). Dirac cones are formed by the first two bands $[11,20]$. The lower and upper Dirac cones at the $K$ point depicted by isofrequency contours, presented in Ref. [20], are reproduced in the same figure [(c) and (d)]. As a matter of fact, departing from the Dirac frequency $\omega_{D} \approx 0.917(\omega a / 2 \pi c)$, the isofrequency lines undergo trigonal distortion and approach quasitriangular forms at $\omega_{1} \approx 0.863$ and $\omega_{2} \approx 0.978(\omega a / 2 \pi c)$ for, respectively, the lower and the upper cones.

The formation of the first two bands can be described by a TB model [11] [solid lines in Fig. 1(b)]. Recalling that, in a such model, the crystal wave function is expressed as the sum of local wave functions, $\left|\phi_{A}\right\rangle$ and $\left|\phi_{B}\right\rangle$, corresponding to the electric field distributions of the $s$-like resonance modes formed inside $A$ and $B$ tiles delimited by three metallic cylinders at the vertices. Along a $\Gamma-K$ direction, the modes $\left|\phi_{A}\right\rangle$ and $\left|\phi_{B}\right\rangle$ contribute equally, in absolute value, to the crystal wave function:

$$
\begin{aligned}
\left|\Psi_{\mathbf{k}}(\mathbf{r})\right\rangle= & \sum_{m} \frac{e^{i \mathbf{k} \mathbf{R}_{m}}}{\sqrt{2 N}}\left(\left|\phi_{A}\left(\mathbf{r}-\mathbf{R}_{m}-\mathbf{d}_{A}\right)\right\rangle\right. \\
& \left. \pm\left|\phi_{B}\left(\mathbf{r}-\mathbf{R}_{m}-\mathbf{d}_{B}\right)\right\rangle\right),
\end{aligned}
$$

where $\mathbf{R}_{m}, \mathbf{d}_{A}$, and $\mathbf{d}_{B}$ represent, respectively, the lattice vectors and the positions of $\left|\phi_{A}\right\rangle$ and $\left|\phi_{B}\right\rangle$ in the unit cell, with $\sum_{m}$
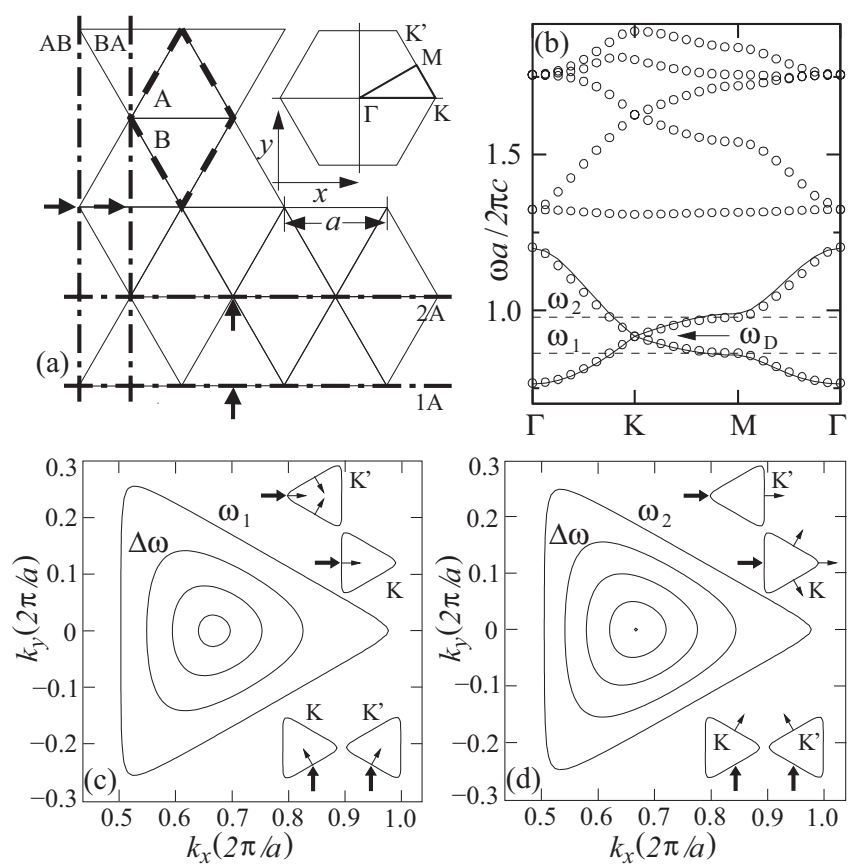

FIG. 1. The triangular lattice and its rhombic unit cell (thick dashed lines) [(a)] of the metallic photonic crystal and the corresponding EM frequency band diagram [(b)], where a Dirac point at $K(4 \pi / 3 a, 0)$ is obtained for $\omega_{D} \approx 0.917$. The circles and solid lines represent, respectively, the FDTD solution and the TB model description. Isofrequency contours of the lower and upper cone at the same $K$ point, with contour interval $\Delta \omega=0.015$, are depicted in (c) and (d). Quasitriangular forms are obtained at frequency levels $\omega_{1} \approx 0.863$ and $\omega_{2} \approx 0.978$, indicated by the dashed lines in (b) (all the values are normalized to $\omega a / 2 \pi c$ ). The upper and lower insets in (c) and (d) depict the expected beam propagation directions (thin arrows) for, respectively, the armchair and zigzag edge incidence (thick arrows). The incidence edges investigated in the present work are depicted by the thick dash-dotted lines in (a), with the thick arrows indicating the incident beam axes.

summing over the unit cells. The wave function is characterized by a mirror or a mirror inversion symmetry with respect to the plane defined by the $\Gamma-K$ line and the $z$ axis.

Defining the usual parameters, $\alpha, \gamma$, and $\beta$, that describe, respectively, the overlap and the energy transfer between first neighbor tiles and the mode energy level shift from that in an individual tile [21], the crystal frequency $\omega_{k}$ can be obtained from the characteristic equation [11]

$$
\operatorname{det}\left[\begin{array}{cc}
\omega_{0}^{2}-\beta-\omega_{k}^{2} & -\xi\left[\gamma-\alpha\left(\omega_{0}^{2}-\omega_{k}^{2}\right)\right] \\
-\xi^{*}\left[\gamma-\alpha\left(\omega_{0}^{2}-\omega_{k}^{2}\right)\right] & \omega_{0}^{2}-\beta-\omega_{k}^{2}
\end{array}\right]=0
$$

with $\omega_{0}$ the eigenfrequency of the $s$-like resonance modes in an individual triangular tile.

The roots are simply

$$
\omega_{k}^{2}=\omega_{0}^{2}-\frac{\beta \mp|\xi| \gamma}{1 \mp|\xi| \alpha}
$$


with

$$
|\xi|=\left\{1+4\left[\cos \left(\frac{\sqrt{3} k_{y} a}{2}\right) \cos \left(\frac{k_{x} a}{2}\right)+\cos ^{2}\left(\frac{k_{x} a}{2}\right)\right]\right\}^{\frac{1}{2}}
$$

The value of $\omega_{0}$ can be obtained by solving Maxwell's equation using the FDTD method on an individual triangular tile.

As shown in Figs. 1(c) and 1(d), along the $x$ axis, the left edges of the quasitriangular contours are close to the wave vector $k=(\pi / a, 0)$. This relation is valid for the other $\Gamma-K$ directions by rotational symmetry, where the edges of the quasitriangular contours inside the first Brillouin zone are close to $k=(-\pi / a, 0)$ and $( \pm \pi / 2 a, \pm \sqrt{3} \pi / 2 a)$, respectively. Indeed, one can check that the magnitude differences between the concerned contour edge wave vectors and the above $k$ vectors are less than $1 \%$. Therefore, wave vectors close to the edges of the quasitriangular contours are close to the above $k$ vectors as well. For these wave vectors, the sum $\cos \left(\frac{\sqrt{3} k_{y} a}{2}\right) \cos \left(\frac{k_{x} a}{2}\right)+\cos ^{2}\left(\frac{k_{x} a}{2}\right)$ in Eq. (4) tends to zero, and Eq. (3) can be rewritten as

$$
\omega_{k}^{2} \approx \Omega_{0}^{2}-\frac{\mp 2\left[\cos \left(\frac{\sqrt{3} k_{y} a}{2}\right) \cos \left(\frac{k_{x} a}{2}\right)+\cos ^{2}\left(\frac{k_{x} a}{2}\right)\right] \gamma}{1 \mp \alpha}
$$

with

$$
\Omega^{2}=\omega_{0}^{2}-\frac{\beta \mp \gamma}{1 \mp \alpha}
$$

(干 corresponding to the upper and lower Dirac cone, respectively.)

The above relation between the wave vectors can also be checked by considering the corresponding frequency levels. Taking the frequency bands along the $x$ axis, the values of $\Omega$ are exactly the crystal frequency levels at $k=(\pi / a, 0)$, where $|\xi|$ is unity [Eqs. (3), (4), and (6)]. We get from the FDTD solutions $\omega_{(\pi / a, 0)_{1}}=\Omega_{1} \approx 0.8626$ and $\omega_{(\pi / a, 0)_{2}}=$ $\Omega_{2} \approx 0.9792(\omega a / 2 \pi c)$. These values are close to the frequency levels of the quasitriangular contours $\omega_{1}$ and $\omega_{2}$ given above.

As a matter of fact, the $\Omega^{2}$ expression in Eq. (6) corresponds to the energy levels of two coupled modes $\left|\phi_{A}\right\rangle$ and $\left|\phi_{B}\right\rangle$ in a unit cell, of which the long diagonal is perpendicular to the $\Gamma-K$ axis, with $\mp$ corresponding, respectively, to the antibonding and bonding states. The band structure along a $\Gamma-K$ direction close to the quasitriangular contours, associated to the lower or upper parts of the Dirac cones, can then be viewed as arising from a series of $\left|\phi_{A}\right\rangle-\left|\phi_{B}\right\rangle$ bonding or antibonding states on one-dimensional chains, with the band frequencies derived from the $\Omega$ values adjusted by the second term in Eq. (5).

Along a $\Gamma-K$ axis, the $D_{6 h}$ symmetry is lowered to that of a subgroup $C_{2 v}$ [22]. The $\left|\phi_{A}\right\rangle-\left|\phi_{B}\right\rangle$ bonding and antibonding states, as well as the crystal wave function, can be described by the $A_{1}$ and $B_{1}$ representations of $C_{2} V$, characterized by, respectively, a mirror and a mirror inversion symmetry with respect to the $\Gamma-K-z$ plane. An EM beam propagating along a $\Gamma-K$ direction and associated to the lower Dirac cone is described by the $A_{1}$ representation, while such a beam associated to the upper cone is represented by $B_{1}$ [20]. They will be referred to as $A_{1}$ and $B_{1}$ beams hereinafter.
It is worth emphasizing that, in the present structure, a $\left|\phi_{A}\right\rangle-\left|\phi_{B}\right\rangle$ bonding or antibonding state can only be formed in a rhombic unit cell, since the coupling takes place through the opening at the common edge between $A$ and $B$ tiles [see Fig. 1(a)]. An $A_{1}$ or $B_{1}$ beam will then propagate based on these $\left|\phi_{A}\right\rangle-\left|\phi_{B}\right\rangle$ states formed in the unit cells along the $\Gamma-K$ direction. Both of these beams will be characterized by a minimum width that is the height of the unit cell along its long diagonal.

In a metallic photonic crystal, where the coupling is of short range, as described by the TB model, the coupling strength between an incident beam and the first resonance modes excited in the crystal sample is decisive for the transmission, since the magnitudes of these resonance modes determine those of the following along the propagation direction, thus the beam transmission. Moreover, for an EM beam with wave vector close to the quasitriangular isofrequency contours of the Dirac cones, the above discussion suggests the importance for the incident beam to excite $\left|\phi_{A}\right\rangle-\left|\phi_{B}\right\rangle$ bonding or antibonding states, in the first rhombic unit cells, at the sample edges for an optimum transmission. It is therefore interesting to investigate the EM beam propagation for different local incident conditions at the sample edges. This can be achieved using narrow incident beams, of widths of the crystal period sizes, that allow us to selectively interact with specific local structure configurations, to probe the coupling between the incident field and the local resonance modes associated to the Dirac cones at elementary structure scales.

\section{NARROW ELECTROMAGNETIC BEAM TRANSMISSION}

\section{A. Armchair edge incidence}

There are three expected propagation directions for the transmitted beams from the armchair edge, a central beam in the same direction as the incident one, and two side beams at $\pm \pi / 3$ angles relative to the incident direction, associated to the lower and upper parts of the Dirac cones for $K$ and $K^{\prime}$ valleys [Figs. 1(c) and $1(\mathrm{~d})$ ]. The propagation directions are perpendicular to three families of rhombic unit cells [see Fig. 1(a)] that allow the formation of coupled $\left|\phi_{A}\right\rangle-\left|\phi_{B}\right\rangle$ pairs mentioned in Sec. II.

The incident beam can only impinge on entire unit cells that are parallel to the armchair edge, rather than on that at $\pm \pi / 3$ angles. At local scale, there are two tile patterns at this edge, an $A-B$ and a $B$ - $A$ tile pair. Here we consider two sample configurations, both $16 \times 16$ periods in size, characterized by an aspect ratio $\sqrt{3}$ ensuring the same path lengths for the central and side beams. They offer two armchair edges shifted by a half period in the $x$ direction, bringing forth, respectively, an $A-B$ and a $B$ - $A$ tile pair at the edge center [see Fig. 1(a)]. These two cases will be referred to as $A B$ and $B A$ cases hereinafter.

We consider an incident beam of the width of the lattice period along the $y$ axis, $\sqrt{3} a$, that covers the height of a unit cell along its long diagonal direction. The beam is TM polarized and emitted by a line source of length $\sqrt{3} a$, placed at the sample left edge center. For these two cases, the incident beam impinges, respectively, on an $A-B$ and a $B-A$ tile pair. The transmissions are considered in two outgoing regions. 

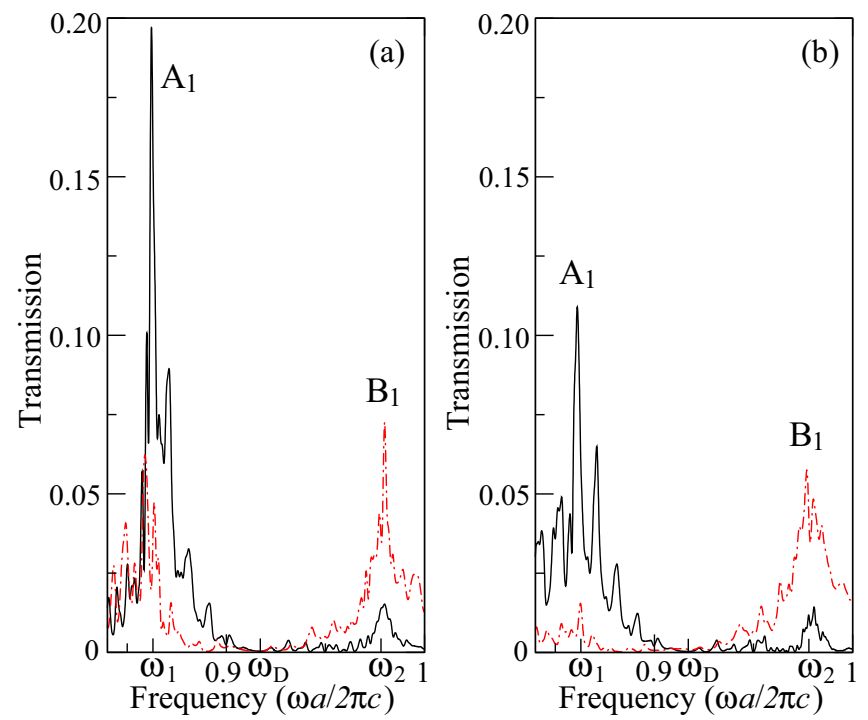

FIG. 2. Transmission spectra for the armchair edge incidence for the $A B[(\mathrm{a})]$ and $B A[(\mathrm{~b})]$ cases in the center (solid lines) and side beam (dash-dotted lines) directions in the vicinity of the Dirac frequency. The maximum transmission peaks correspond to $\omega \approx 0.862$ and 0.980 in (a) and $\omega \approx 0.861$ and $0.977(\omega a / 2 \pi c)$ in (b) for the $A_{1}$ and $B_{1}$ beams, respectively.

One, with the same width $\sqrt{3} a$ as the incident beam, is placed at the opposite side of the sample in front of the incident beam for the central beam direction; the other, with width $2 a$, at the sample upper side (a zigzag edge), at $\pi / 3$ angle relative to the incident direction, for one of the side beams. Here we choose $2 a$ for the side beam direction, since both the bonding and antibonding states in a unit cell span two periods at the zigzag edge. Using an incident Gaussian beam centered at $\omega_{D}$, the transmission spectra, obtained using the FDTD method and normalized to the incident flux, are displayed in Fig. 2. One can see that maximum transmission peaks are obtained at frequency levels close to $\omega_{1}$ and $\omega_{2}$, corresponding, respectively, to the $A_{1}$ and $B_{1}$ beams associated to the lower and upper part of the Dirac cones, along the central and the side directions. Their counterparts, i.e., the transmission peaks for the same beams but along the side and central directions, are much weaker.

Moreover, for the $A B$ case, the central $A_{1}$ beam displays a much stronger transmission peak than the side $B_{1}$ beam, in spite of the non-negligible transmission for the $A_{1}$ beam in the side beam directions. Indeed, the side $B_{1}$ beam transmission peak is only about $35 \%$ that of the central $A_{1}$ beam. The situation is significantly different for the $B A$ case, where the central $A_{1}$ beam displays a much weaker transmission peak that is only about $55 \%$ that of the central $A_{1}$ beam in the $A B$ case, while the transmission peak of the side $B_{1}$ beam, with a difference of only about $20 \%$, is closer to its counterpart in the $A B$ case.

The $A_{1}$ and $B_{1}$ beam propagations at their respective transmission peak frequencies are obtained using the FDTD method, and images of the electric field distributions are displayed in Figs. 3 and 4. These two figures show that, except in the vicinity of the impact zones for the $B_{1}$ beams (this
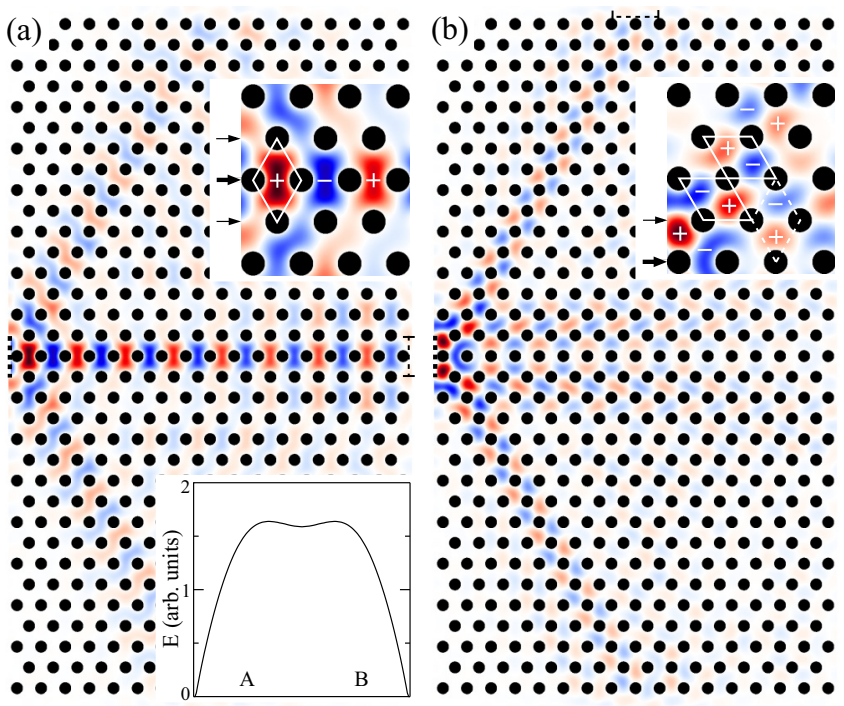

FIG. 3. Electric field distributions of the $A_{1}[(\mathrm{a})]$ and $B_{1}[(\mathrm{~b})]$ beams corresponding to the two maximum transmission peaks in Fig. 2(a) for the $A B$ case, with the thick dashed line at the sample left side symbolizing the line source, and the thin dashed lines at the right and upper sides the outgoing regions for the central and side beam directions. The upper part insets show a magnified region in the vicinity of the impact zone, with the thick and thin arrows indicating the incident beam axes and edges, and the \pm signs the field polarities. The unit cells in the central [(a)] and side beam [(b)] directions are delimited by solid lines, and that related to the $B_{1}$ beam along the incident beam edge in the central direction by dashed lines [(b)]. The lower part inset in (a) shows the electric field magnitude along the long diagonal of the unit cell, passing by the $A$ and $B$ tiles, in the upper inset.

point will be discussed in the following), the electric fields of the $A_{1}$ and $B_{1}$ beams are formed by resonance modes in the unit cells along the propagation directions, with polarities characterized, respectively, by a mirror and a mirror inversion
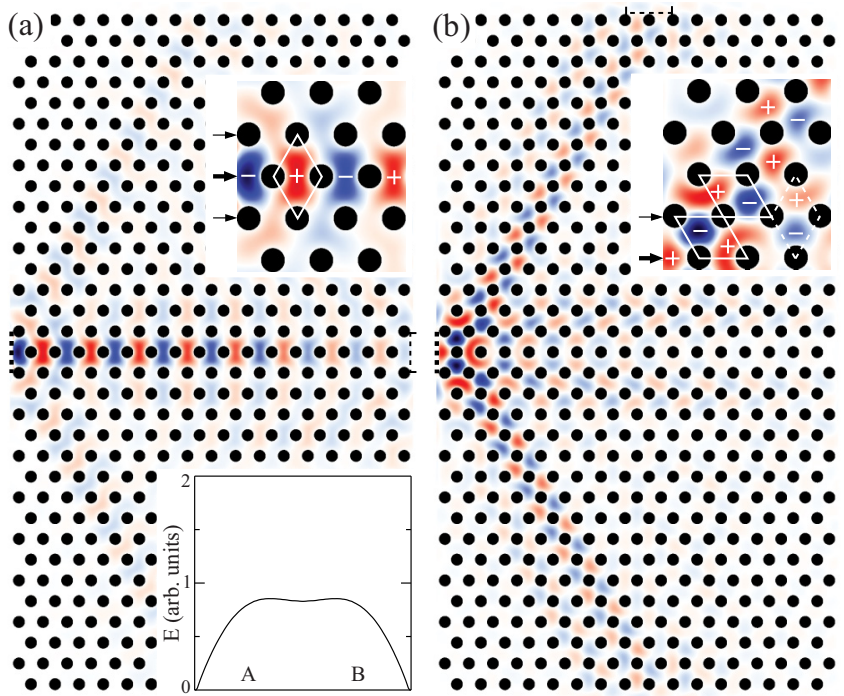

FIG. 4. Same disposition as in Fig. 3, but for the $B A$ case and corresponding to the two maximum transmission peaks in Fig. 2(b). 
symmetry with respect to the plane formed by the propagation axis and the $z$ axis, in agreement with the discussion in Sec. II that the beam propagation can be considered as being based on a series of bonding or antibonding states formed in the unit cells.

Moreover, Figs. 3 and 4 illustrate the relationship between the incident beam and the mode excitation in the two samples. For the $A B$ case, as shown in Fig. 3(a), the incident beam can excite an $A_{1}$ beam of minimum width, that corresponds to the unit cell height as mentioned in Sec. II, immediately from the impact zone. Indeed, the incident beam impinges on a unit cell formed by an $A-B$ tile pair, and a bonding state is formed in the impacted unit cell through the coupling between the $\left|\phi_{A}\right\rangle$ and $\left|\phi_{B}\right\rangle$ modes in the two tiles, as shown in the upper inset of Fig. 3(a). The $A_{1}$ beam can be considered as being directly coupled to the incident field.

In the $B A$ case, on the contrary, the incident beam cannot excite directly the same $A_{1}$ beam, like in the $A B$ case, in the immediate impact zone. In fact, here the beam impinges on a $B$-A tile pair, in which a bonding state between $\left|\phi_{B}\right\rangle$ and $\left|\phi_{A}\right\rangle$ modes cannot be formed due to the lack of coupling, as discussed in Sec. II. Indeed, as shown in the upper inset of Fig. 4(a), such a state is formed further inside the sample, in the rhombic unit cell situated behind the $B-A$ pair. The coupling between the incident beam and the $A_{1}$ beam can be considered as being indirect by comparison with the $A B$ case.

In order to further probe the coupling effect between the incident field and the resonance modes, let us consider the electric field distribution of the $\left|\phi_{A}\right\rangle-\left|\phi_{B}\right\rangle$ bonding state in the first unit cell in front of the incident beam, at the beginning of the transmitted $A_{1}$ beam of, respectively, the two cases. The field distributions along the long diagonal of the unit cells are displayed in the lower insets of Figs. 3(a) and 4(a). These figures show that, for the same incident beam, the field magnitude in the first unit cell in the $A B$ case is about $90 \%$ lager than that in the $B A$ one, confirming that the coupling between the incident field and the $A_{1}$ beam is more efficient in the $A B$ case. Moreover, these figures confirm that the field distributions in these two cases display a mirror symmetry with respect to the $x z$ plane and can indeed be considered as bonding states formed by a pair of $\left|\phi_{A}\right\rangle$ and $\left|\phi_{B}\right\rangle$ modes.

Concerning the side $B_{1}$ beams in both cases, their weak transmissions can be related to the local field distributions created by the incident beams. Indeed, due to the orientations and positions of the unit cells along the $B_{1}$ beam propagation directions, antibonding states cannot be directly excited by the incident fields in the first unit cells at the beginning of the propagation paths. In fact, as shown in Figs. 3(b) and 4(b), the resonances modes are irregularly distributed in the vicinity of the impact zones, with some of them extending outside the tiles. The local fields in the first unit cells cannot be considered as antibonding states between $\left|\phi_{A}\right\rangle$ and $\left|\phi_{B}\right\rangle$ pairs, and they lack clearly the mirror inversion symmetry with respect to the plane defined by the $B_{1}$ beam axis and the $z$ axis (we will show below that this can also be seen in terms of the electric field magnitudes in the unit cells). However, thanks to their polarity distributions, with opposite electric field polarities in the two tiles of the unit cells, these modes can be constructively coupled to the antibonding states on the

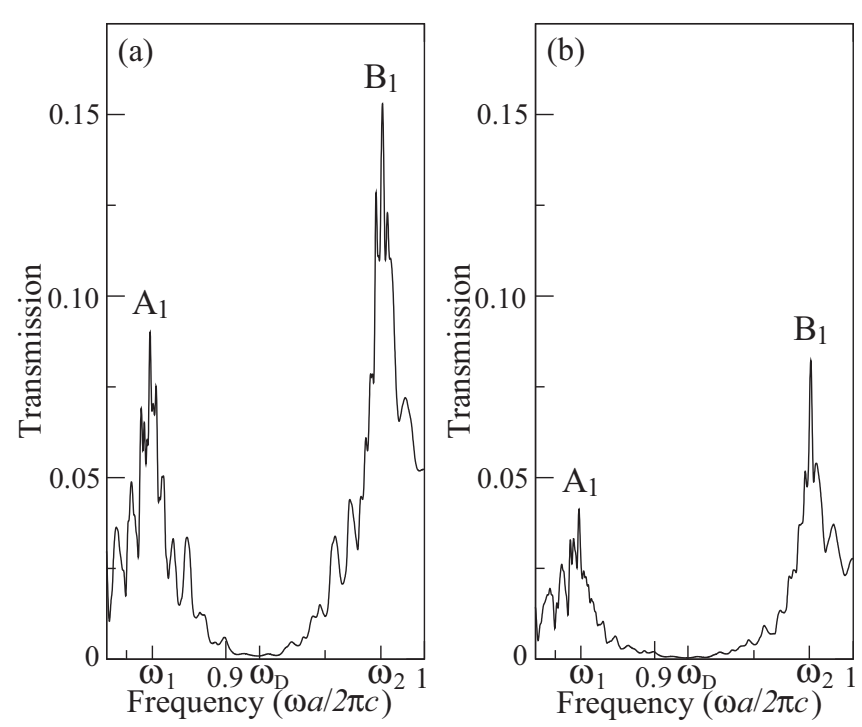

FIG. 5. Transmission spectra for the zigzag edge incidence for the $1 A[(\mathrm{a})]$ and $2 A[(\mathrm{~b})]$ cases in the vicinity of the Dirac frequency. The maximum transmission peaks correspond to $\omega \approx 0.862$ and $0.979(\omega a / 2 \pi c)$ for, respectively, the $A_{1}$ and $B_{1}$ beams in both cases.

propagation paths. Besides, as we can see in Figs. 2, 3(b), and 4(b), although the $B_{1}$ beams are inhibited along the central axis of the incident beams, due to the incompatibility between the mirror symmetry of the latter and the mirror inversion symmetry of the former [20], there is weak transmission in the central direction along the incident beam edges. This can be attributed to mode excitations at the edges of the incident beams [see the insets in Figs. 3(b) and 4(b)], where the latter do not have mirror symmetry. The local fields formed at the sample edges, where the latter intercept the beam edges, display opposite polarities along the $y$ axis, due to the irregularly extended spatial distribution of certain modes, and lead to $B_{1}$ beams propagating in the central direction (see the unit cells delimited by dashed lines in the above figures). These points will be further discussed in the following.

\section{B. Zigzag edge incidence}

There are two expected propagation directions for the transmitted beams from the zigzag edge, at, respectively, $\pm \pi / 6$ angles relative to the incident direction, associated to the lower and upper parts of the Dirac cones for $K$ and $K^{\prime}$ valleys [Figs. 1(c) and 1(d)] and perpendicular to two families of rhombic unit cells [see Fig. 1(a)]. All the transmitted beams are symmetrically compatible with an incident beam, since two transmitted beams together, either two $A_{1}$ or two $B_{1}$ ones, are described by a mirror symmetry with respect to the $y z$ plane. For this orientation, an incident beam can only impinge on the $A$ tiles. It can excite directly neither bonding nor antibonding $\left|\phi_{A}\right\rangle-\left|\phi_{B}\right\rangle$ pair states in the first unit cells at the beginning of the propagation paths.

We consider two sample configurations, both $32 \times 8$ periods in size, offering two zigzag edges shifted by a half period in the $y$ direction, bringing forth, respectively, an $A$ tile and an $A-A$ tile pair at the low edge center [see Fig. 1(a)]. These two cases will be referred to as $1 A$ and $2 A$ cases hereinafter. 
(a) $\bullet \bullet \bullet \bullet \bullet \bullet \bullet \bullet \bullet \bullet \bullet \bullet \bullet \bullet \bullet \bullet \bullet \bullet \bullet-b^{-b} \bullet \bullet \bullet \bullet \bullet \bullet \bullet$ a) $\bullet \bullet \bullet \bullet \bullet \bullet \bullet \bullet \bullet \bullet \bullet \bullet \bullet \bullet \bullet \bullet \bullet \bullet \bullet \bullet \bullet \bullet \bullet \bullet \bullet \bullet \bullet \bullet \bullet \bullet \bullet \bullet \bullet \bullet$ $\because \bullet \bullet \bullet \bullet \bullet \bullet \bullet \bullet \bullet \bullet \bullet \bullet \bullet \bullet \bullet \bullet \bullet \bullet \bullet \bullet \bullet \bullet \bullet \bullet \bullet \bullet \bullet \bullet \bullet$ $\bullet \bullet \bullet \bullet \bullet \bullet \bullet \bullet \bullet \bullet \bullet \bullet \bullet \bullet \bullet \bullet \bullet \bullet \bullet \bullet \bullet \bullet \bullet \bullet \bullet \bullet \bullet \bullet \bullet \bullet$ $\bullet \bullet \bullet \bullet \bullet \bullet \bullet \bullet \bullet \bullet \bullet \bullet \bullet \bullet \bullet \bullet \bullet \bullet \bullet \bullet \bullet \bullet \bullet \bullet$ ( $10 \bigcirc$

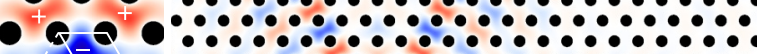
$\theta \Rightarrow \rightarrow$

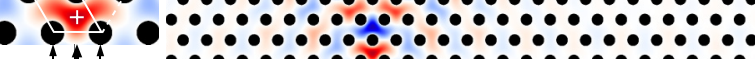
$\bullet \bullet \bullet \bullet \bullet \bullet \bullet \bullet \bullet \bullet \bullet \bullet \bullet \bullet \bullet \bullet \bullet \bullet \bullet \bullet \bullet \bullet \bullet$

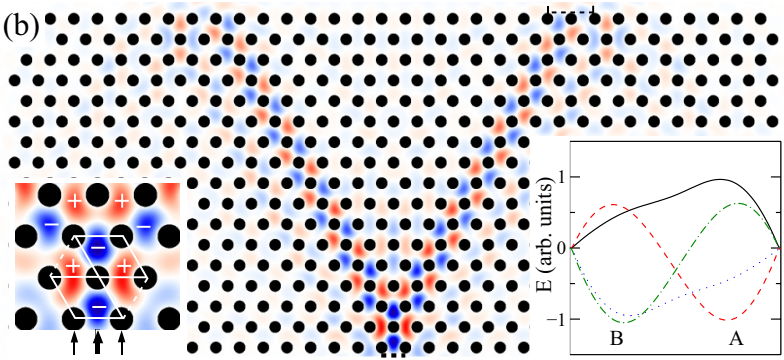

FIG. 6. Electric field distributions of the $A_{1}[(\mathrm{a})]$ and $B_{1}[(\mathrm{~b})]$ beams corresponding to the two maximum transmission peaks in Fig. 5(a) for the $1 A$ case, with the thick dashed line at the sample lower side symbolizing the line source, and the thin dashed line at the upper side the beam outgoing region. The left part insets show a magnified region in the vicinity of the impact zone, with the thick and thin arrows indicating the incident beam axes and edges, and the \pm signs the field polarities. The right part inset in (b) shows the electric field magnitudes along the long diagonal, passing by the $B$ and $A$ tiles, of the first and the second unit cells outlined by solid lines in the left insets in, respectively, (a) (solid and dotted lines) and (b) (dashed and dash-dotted lines). A hexagon can be defined by the two unit cells connected by the dashed lines.

An incident beam with width $a$, covering one period along the $x$ axis, is considered. The beam is TM polarized and emitted by a line source of length $a$, placed at the sample lower edge center, and impinging, respectively, on an $A$ tile and two half $A$ tiles. The transmission is considered in an outgoing region with width $2 a$ (as mentioned above, both the bonding and antibonding states in a unit cell span two periods at the zigzag edge) at $\pi / 6$ angle at the sample upper side. For both cases, an incident Gaussian beam centered at $\omega_{D}$ is used. The transmission spectra, obtained using the FDTD method and normalized to the incident flux, are displayed in Fig. 5.

Figure 5 shows that, like in the cases of the armchair edges, maximum transmission peaks are obtained at frequency levels close to $\omega_{1}$ and $\omega_{2}$, corresponding, respectively, to the $A_{1}$ and $B_{1}$ beams associated to the lower and upper part of the Dirac cones. The beam propagation in these samples, for monochromatic waves at the transmissions peak frequencies, is obtained using the FDTD method, and images of the electric field distributions are displayed in Figs. 6 and 7. Like for the armchair incidence, these two figures are in agreement with the discussion in Sec. II, except in the vicinity of the impact zones, that the beam propagation can be considered as being based on a series of bonding or antibonding states in the unit cells.

Concerning the magnitudes of the beam transmission, Fig. 5 shows that, contrary to the armchair edge cases, here
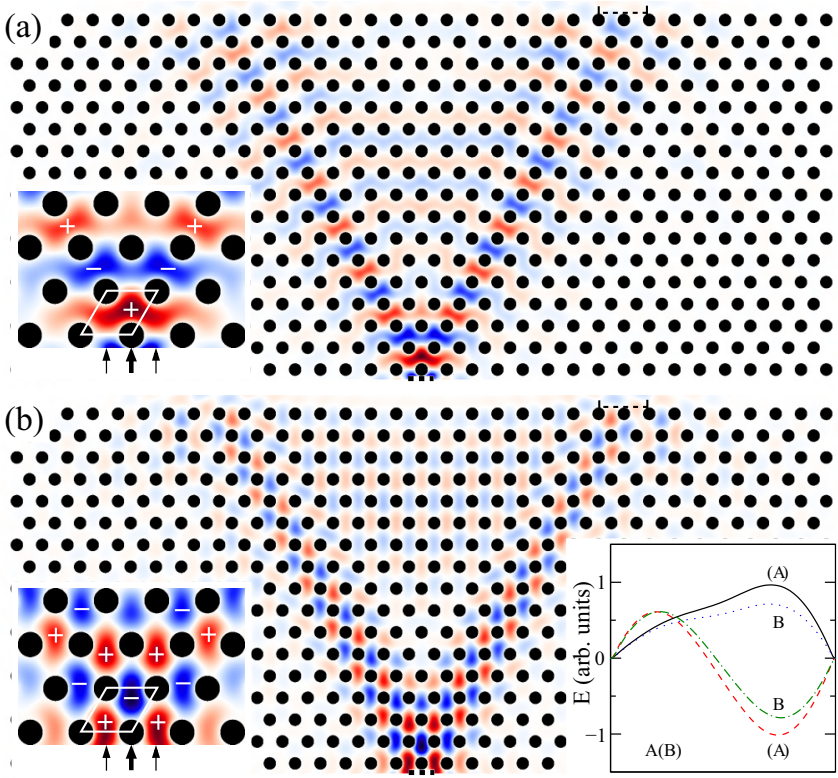

FIG. 7. Same disposition as in Fig. 6, except that it concerns the $2 A$ case, with the beams corresponding to the two maximum transmission peaks in Fig. 5(b), and that the right part inset in (b) displays the electric field magnitudes along the long diagonal, passing by the $A$ and $B$ tiles, of the first unit cell outlined by solid lines in the left insets in, respectively, (a) (dotted line) and (b) (dash-dotted line), together with that passing by the $B$ and $A$ (bracketed letters in the figure) tiles in the first unit cell of the $1 A$ case in Figs. 6(a) (solid line) and 6(b) (dashed line).

the $B_{1}$ beam transmission peaks display stronger magnitudes than that of the $A_{1}$ beams for both the cases. Indeed, for the $1 A$ case, the maximum transmission peak of the $B_{1}$ beam is about $70 \%$ higher than that of the $A_{1}$ beam, while, for the $2 A$ case, the maximum transmission peak of the $B_{1}$ beam is almost twice that of the $A_{1}$ one. Moreover, in the latter case, the transmission peaks of both $A_{1}$ and $B_{1}$ beams are much lower compared to that in the $1 A$ case, corresponding, respectively, to about $45 \%$ and $55 \%$ that of their counterparts.

As pointed out above, neither bonding nor antibonding pair states can be excited directly at the zigzag edge; the beam transmissions can be investigated in terms of the coupling effect that can be probed through both the spatial and magnitude distributions of the excited local fields. Indeed, for both the $A_{1}$ and $B_{1}$ beams, the local fields in the unit cells at the beginning of the propagation paths (left insets in Figs. 6 and 7) cannot be considered as bonding or antibonding states between $\left|\phi_{B}\right\rangle-\left|\phi_{A}\right\rangle$ pairs, though they can be constructively coupled to such states. Moreover, for both beams in the $1 A$ case, the local fields are confined in a hexagon formed by six tiles in the vicinity of the impact zone, while, in the $2 A$ case, the fields are more irregularly distributed, with certain modes extending far out of the tiles.

As far as the local field magnitudes are concerned, the $B_{1}$ beams involve stronger fields than the $A_{1}$ beams in both cases. This can be seen on the field magnitude distributions along the long diagonal of the unit cells in the right insets of Figs. 6(b) and 7(b). In the $1 A$ case, the field magnitudes in the 
$B$ and $A$ tiles of the second unit cell are about $10 \%$ and $27 \%$ larger for the $B_{1}$ beam than for the $A_{1}$ one. In the $2 A$ case, the magnitudes are about $10 \%$ and $32 \%$ larger in the $A$ and $B$ tiles of the first unit cell for the $B_{1}$ beam than for the $A_{1}$ one. This is in agreement with stronger $B_{1}$ beam transmission in both cases.

It is interesting to further compare the coupling between the incident fields and the local modes in the first unit cells, for, respectively, the $A_{1}$ and $B_{1}$ beams, between the $1 A$ and $2 A$ cases. In fact, as shown in the right inset of Fig. 7(b), for the $A_{1}$ beams, the field magnitudes along the cell long diagonal in the left and right tiles are about $13 \%$ and $35 \%$ stronger in the $1 A$ case as compared to the $2 A$ one; while, for the $B_{1}$ beams, the field magnitudes are almost the same in the left tiles for the two cases, and that in the right tile in the $1 A$ case is about $30 \%$ stronger than that in the $2 A$ case. This shows clearly a stronger coupling for both $A_{1}$ and $B_{1}$ beams in the $1 A$ case, leading to stronger transmissions.

The right inset of Fig. 7(b) shows also that, for both $1 A$ and $2 A$ cases, the fields in the first unit cells have stronger magnitudes in the right tiles that are just the first tiles on the incident beam axes at the edge center (respectively, an $A$ and a $B$ tile for the $1 A$ and $2 A$ cases), and it is basically in these tiles that the stronger coupling with the incident fields is manifested for the two beams of the $1 A$ case.

This can be related to the nature of the local fields and their coupling with the incident beams. As a matter of fact, in the $1 A$ case, the field distributions in the hexagon for the $A_{1}$ and $B_{1}$ beams can be considered as corresponding, respectively, to a bonding and an antibonding state formed by the six resonance modes on the hexagonal ring, similar to that of the $\pi$ molecular orbitals of benzene. A description of this classic case can be found in Ref. [23], where the two states are labeled as $\phi_{2, \text { real }}$ and $\phi_{4, \text { real }}$. Indeed, these field distributions have a mirror symmetry with respect to the $y z$ plane, and, as shown in the right inset in Fig. 6(b), for each beam, the modes in the two tiles (an $A$ and a $B$ tile) on the incident beam axis have almost the same magnitudes, that are roughly twice larger than that in the other four tiles of the hexagon (with a factor of about 1.9 and 1.7 for the $A_{1}$ and $B_{1}$ beams). The formation of these states is not surprising, since their frequency levels, resulting from the coupling between neighbor modes on the hexagonal ring, correspond, respectively, to that of the bonding and antibonding states formed by a single pair of neighbor modes. They can thus be excited by incident beams with frequencies close to $\omega_{1}$ and $\omega_{2}$ impinging on the central $A$ tile. While, in the $2 A$ case, the incident beams cannot be coupled to such states, because the incident beam axis passes between two $A$ tiles rather than by an $A$ tile center. As can be seen in the right inset of Fig. 7(b), the magnitudes of the modes in the $A$ tile of the unit cell, for both $A_{1}$ and $B_{1}$ beams, correspond roughly to half that in the central $A$ tile for their counterparts in the $1 A$ case, since here the incident beams impinge on two half $A$ tiles, the coupling is thus weaker for each of these modes. The weaker magnitudes of the modes in the central $B$ tile for the two beams, compared to that in the central $A$ tile in the $1 A$ case, can be attributed to the fact that the modes in the $B$ tile are only indirectly coupled to the incident beams, through the coupling with the modes in the two adjacent $A$ tiles.

\section{DISCUSSION}

The above results underline the importance of the coupling condition for the incident field and the resonance modes at local scales in the beam transmission, associated to the Dirac cones for different $K$ valleys. Indeed, due to the metallic nature of the crystal, as well as the frequency band structure in the vicinity of the quasitriangular Dirac cone contours, a good transmission necessitates, for an incident field, strong coupling with the local resonance modes, as well as the excitation of resonance mode distributions corresponding to bonding or antibonding states in the first unit cells. These aspects are determined by the unit cell exposition and orientation with respect to the incident beam. This point can further be probed by local structure modification that alters the coupling with the incident beam.

Let us consider the $A B$ case, where the side $B_{1}$ beam transmission is much weaker than that of the central $A_{1}$ beam. As mentioned in Sec. III A, antibonding states can not be directly excited by the incident beam in the unit cells at the beginning of the $B_{1}$ beam paths. The resonance modes are irregularly distributed in the vicinity of the impact zone. Moreover, the mode excitation at the beam edges leads to transmission in the central direction. However, by creating a structure vacancy by removing the cylinder at the armchair edge center, i.e., on the incident beam axis, we get the transmission spectra in Fig. 8(a) for the same incidence. The transmission peaks for the $A_{1}$ and $B_{1}$ beams now have similar magnitudes. Indeed, comparing to the original $A B$ case in Fig. 2(a), the $A_{1}$ beam transmission peak is decreased by about $30 \%$, while the $B_{1}$ beam transmission peak is increased by about $80 \%$.

This can be related to the change in the coupling condition. As a matter of fact, the decrease of the $A_{1}$ beam transmission can straightforwardly be attributed to the fact that the incident beam, with frequency close to $\omega_{1}$, can no more be directly coupled to a bonding state, since it now impinges on a $B-A$ tile pair, like in the $B A$ case. The transmission is stronger (by about $25 \%$ ) compared to the $A_{1}$ beam in the latter case, because the incident beam has a lager aperture, the two cylinders at the extremity of the line source being at $a / 2$ away.

Let us focus on the $B_{1}$ beam transmission. The electric field distribution corresponding to the $B_{1}$ beam is displayed in Fig. 8(b). As a matter of fact, the $B_{1}$ beam transmission peak increase can be attributed to the improved coupling condition for the incident beam with frequency close to $\omega_{2}$ that leads to field distribution close to antibonding states in the vicinity of the impact zone. Indeed, the removal of the central cylinder leaves exposed locally two portions of armchair edges at $\pm \pi / 3$ angles, allowing a better exposition of the first unit cells, at the beginning of the beam propagation paths, to the incident beam, without the latter being scattered by the central cylinder, ensuring thus a better coupling between the incident field and the resonance modes in these unit cells. This can be seen in the upper inset of Fig. 8(b) that shows that the resonance modes in the vicinity of the impact zone display more regular spatial distributions as compared to the original $A B$ case in Fig. 3(b), and, moreover, they are now all confined inside the triangular tiles of the unit cells along the propagation path. As far as the field magnitudes are concerned, the magnitude distribution [lower inset of Fig. 8(b)] along the 


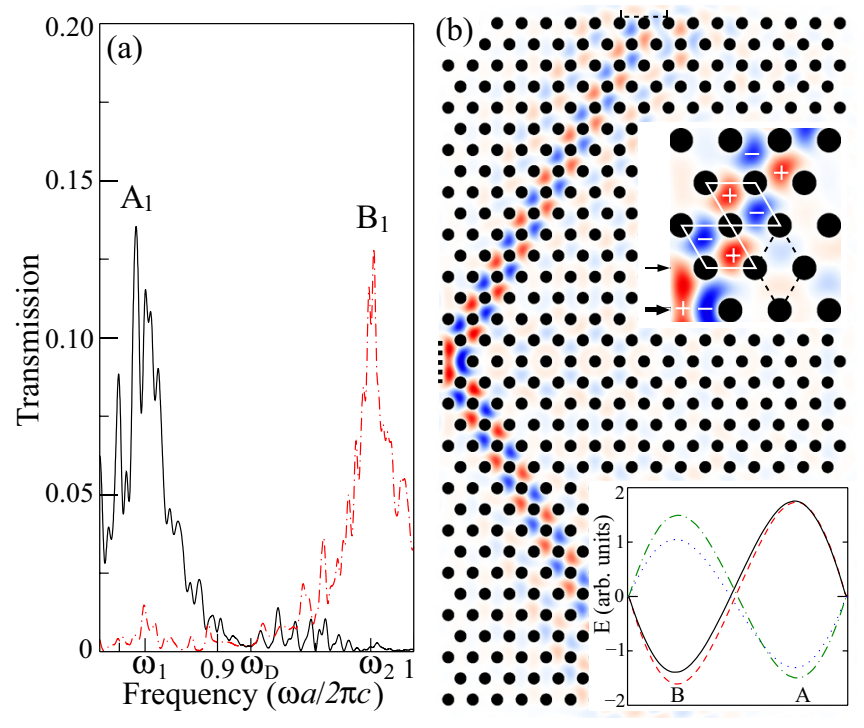

FIG. 8. Transmission spectra for the modified $A B$ case in the center (solid lines) and side beam (dash-dotted lines) directions in the vicinity of the Dirac frequency, with the maximum transmission peaks at $\omega \approx 0.859$ and $0.980(\omega a / 2 \pi c)$ for, respectively, the $A_{1}$ and $B_{1}$ beams [(a)]. The electric field distribution of the $B_{1}$ beam corresponding to the maximum transmission peak in the side beam direction [(b)], with the thick dashed line at the sample left side symbolizing the line source and the thin dashed line at the upper side the outgoing region for the side beam direction. The upper part inset in (b) shows a magnified region in the vicinity of the impact zone, with the thick and thin arrows indicating the incident beam axis and edge, and the \pm signs the field polarities. The unit cells in the side beam direction are delimited by solid lines, and that related to the $B_{1}$ beam along the incident beam edge in the central direction by dashed lines. The lower part inset shows the electric field magnitude along the long diagonal, passing by the $A$ and $B$ tiles, of the first (dashed line) and second (dash-dotted line) unit cells along the propagation direction outlined in the upper inset, together with that in the first (solid line) and second (dotted line) unit cells of the original $A B$ case in Fig. 3(b).

long diagonal of the first unit cell at the beginning of the beam path shows that, as compared to the original $A B$ case, where the mode magnitudes display strong difference, with that in the $B$ tile about $21 \%$ weaker than that in the $A$ tile, the mode in the $B$ tile in the modified case has its magnitude increased, along with the confinement of its spatial distribution. The two modes in the first unit cell have now closer magnitudes, with that in the $B$ tile only about $7 \%$ weaker than that in the $A$ tile (the latter remains almost the same as its counterpart in the original $A B$ case), approaching the field distribution of a $\left|\phi_{B}\right\rangle-\left|\phi_{A}\right\rangle$ antibonding state.

On the other hand, the field distributions at the sample edge, where the latter intercepts the incident beam edges, have no more opposite polarities along the $y$ axis. Indeed, in the original $A B$ case, as shown in the upper inset of Fig. 3(b), the local bipolarlike field distribution at the sample edge is formed due to the irregular spatial distribution of the mode in the $B$ tile in the first unit cell at the beginning of the beam path that extends to the zone on the sample edge above the beam edge axis (the thin arrow). In the modified case, on the contrary, all
TABLE I. Coupling coefficient $k_{c}$ between the incident field and the excited eigenstate (or close to eigenstate) in the first unit cell in several cases, with mod. standing for modified and $b$. and $a . b$. for bonding and antibonding.

\begin{tabular}{lcccc}
\hline \hline Case & $A B$ & $B A$ & $A B$ & (mod.) \\
\hline State & $b$. & $b$. & $b$. & $a . b$. \\
$k_{c}$ & 0.562 & 0.254 & 0.252 & 0.238 \\
\hline \hline
\end{tabular}

the modes are well confined inside the triangular tiles, and, as shown in the upper inset of Fig. 8(b), the field is vanishingly weak in the same zone. This disfavors the mode excitations at the incident beam edges that lead to beam propagation in the central direction mentioned in Sec. III A. Indeed, as shown by the transmission and the field distribution in Fig. 8 , the $B_{1}$ beam transmission in the central direction is almost absent. This can further be confirmed by the field magnitude distributions [lower inset in Fig. 8(b)] in the second unit cell from the beginning of the path [upper insets in Figs. 3(b) and 8(b)]. The field magnitudes are stronger for both modes in the $B$ and $A$ tiles in the modified case, with, respectively, an increase of about $42 \%$ and $14 \%$ as compared to the original case. This can be attributed to the quasiabsence of the transmission in the central direction. Moreover, with a magnitude difference of only about $0.3 \%$ between the modes in the $B$ and $A$ tiles, the field in this unit cell is almost that of a pure $\left|\phi_{B}\right\rangle-\left|\phi_{A}\right\rangle$ antibonding state. This example confirms that the transmission of a narrow incident beam is favored by incident configurations allowing the excitation of resonance modes closest to bonding or antibonding states in the first unit cells, as well as strong coupling between the incident field and the excited modes.

In the case where a $\left|\phi_{B}\right\rangle-\left|\phi_{A}\right\rangle$ bonding or antibonding eigenstate (or a state close to such states) is formed in the first rhombic unit cell at the beginning of the beam propagation path, it is possible to consider the coupling coefficient $k_{c}$ that describes the percentage of the energy transferred from the incident field to this state, in relation to the corresponding beam transmission. This is the situation for the $A_{1}$ beam in both $A B$ and $B A$ cases, and for the $A_{1}$ and $B_{1}$ beams in the modified $A B$ case. $k_{c}$ can be numerically obtained by calculating the flux flowing into the corresponding unit cell and normalizing it to that of the incidence. The obtained $k_{c}$ values in the above cases are listed in Table I. We note that, for the $A B$ and $B A$ cases, the two $k_{c}$ values scale with the two $A_{1}$ beam transmissions, and that, for the modified $A B$ case, the two $k_{c}$ values scale with the transmissions of the $A_{1}$ and $B_{1}$ beams as well. The transmissions of the two latter beams are both stronger than that of the $A_{1}$ beam in the $B A$ case, for comparable $k_{c}$ values. This can be attributed to the larger incidence aperture in the modified $A B$ case already mentioned above. The above results allow us to illustrate the variation of the coupling between the incident field and the resonance modes excited in the first unit cell following different local structure configurations and to better understand the relation between the coupling at local scale and the corresponding beam transmission.

It is worth pointing out that the results concerning the armchair edge in the present investigation are comparable to that in the previous work [20]. The quantitative differences 
in the transmission values can be attributed to two factors. On one hand, the previous work involves a much larger incident beam width, and the transmission should be considered as resulting from a combination of the $A B$ and $B A$ cases of the present work. On the other hand, the metallic waveguide used in the previous work prevents the incident beam spreading in the impact zone and thus reinforces the transmission.

Metallic waveguides are not used in the present study since they will induce significant transmission differences at this scale, modifying the magnitude relations between the transmission peaks. Consider the waveguides of the widths of the crystal periods. In the configurations where the ends of the metallic walls are placed near the sample edge between two neighbor metallic cylinders, the waveguide will reduce, in the $A B$ case, the transmission of both $A_{1}$ and $B_{1}$ beams in the side directions and reinforce artificially the $A_{1}$ beam transmission in the central direction, increasing the ratio between the transmission of the $A_{1}$ beam in the central direction and that of the $B_{1}$ beam in the side directions, while, in the $2 A$ case, a metallic waveguide will drastically reduce the transmissions of both beams, due to the weak opening width remaining between the central cylinder and the wall ends. In the $B A$ and $1 A$ cases, on the contrary, the transmission of the $A_{1}$ and $B_{1}$ beams will both be reinforced. Line sources of sizes of the crystal periods allow us to avoid such problems. Indeed, although their emitted wave fronts do not have flat forms, placed close to the sample edges, such line sources enable probing the coupling at local structure scales by selectively exciting specific local eigenstates in the impact zones. Their sizes allow us to accommodate the finite metallic cylinder diameter. For smaller sized sources, the transmissions will be sensitive to the cylinder reflection effect at the sample edges. Consider, for instance, a point source. In the configurations where the source is placed in front of the central cylinder, i.e., in the $A B$ and $2 A$ cases, both the $A_{1}$ and $B_{1}$ beams will have their transmission drastically reduced due to the central cylinder reflection. Moreover, the ratio between the transmission of the $A_{1}$ beam in the central direction and that of the $B_{1}$ beam in the side directions will be reduced in the $A B$ case, due to the fact that the incident field will be more strongly reflected by the same cylinder in the central direction, while, in the $B A$ and $1 A$ cases, the transmission of both $A_{1}$ and $B_{1}$ beams will be increased since the incident fields will have larger apertures. All this will prevent the comparison of beam transmissions for different incident conditions.

The present investigation allows us to understand the interaction between an incident EM beam and the resonance states, associated to both the lower and upper parts of the Dirac cones for different $K$ valleys, at elementary structure scales reaching the size of these resonance states. It provides information on the relationship between the local structure configurations and the transmission of an incident EM beam, with wave vector close to the quasitriangular isofrequency contours of the Dirac cones, at both the armchair and zigzag edges, and may find application in valley photonics for tuning and optimizing the beam transmission in the design of various beam collimation and splitting devices to convey information and address different valleys. Moreover, since this investigation deals with EM beam width at its minimum limit for a metallic photonic crystal, its results can be of specific interest to miniaturized low-power photonic systems.

\section{CONCLUSION}

This investigation demonstrates the key role of the coupling between the local resonance modes, at both the armchair and zigzag edges, and an incident EM beam, with wave vector close to the quasitriangular isofrequency contours of the Dirac cones, in the beam transmission in a graphenelike triangular metallic photonic crystal. Indeed, by probing the coupling at elementary structure scales with narrow incident EM beams, it shows strong excitation difference in the first unit cells, following their positions and orientations with respect to the incident fields. This in turn determines the beam transmission associated to the upper and lowers parts of the Dirac cones for different $K$ valleys. Large transmission is obtained for incident conditions allowing strong coupling between the incident fields and the resonance modes in the first unit cells at the incident edges and leading to field distributions corresponding or close to bonding or antibonding states for these modes. This work allows understanding of the particularity of the graphenelike metallic photonic crystal in its interaction with the EM waves at scales down to the size of local resonance states. It may find application in valley photonics in the designs of beam collimation and splitting devices for information processing, by providing information for tuning and optimizing the beam transmission in various conditions.

\section{ACKNOWLEDGMENT}

The author wishes to acknowledge the anonymous referee for the suggestion of evaluating the coupling coefficient between the incident field and the excited eigenstates in relation to the beam transmissions.
[1] O. Peleg, G. Bartal, B. Freedman, O. Manela, M. Segev, and D. N. Christodoulides, Phys. Rev. Lett. 98, 103901 (2007).

[2] R. A. Sepkhanov, Ya. B. Bazaliy, and C. W. J. Beenakker, Phys. Rev. A 75, 063813 (2007).

[3] S. Raghu and F. D. M. Haldane, Phys. Rev. A 78, 033834 (2008).
[4] X. Huang, Y. Lai, Z. H. Hang, H. Zheng, and C. T. Chan, Nat. Mater. 10, 582 (2011).

[5] J. L. Garcia-Pomar, A. Cortijo, and M. Nieto-Vesperinas, Phys. Rev. Lett. 100, 236801 (2008).

[6] A. Rycerz, J. Tworzydlo, and C. W. J. Beenakker, Nat. Phys. 3, 172 (2007). 
[7] J.-W. Dong, X.-D. Chen, H. Zhu, Y. Wang, and X. Zhang, Nat. Mater. 16, 298 (2017).

[8] T. Ma and G. Shvets, New J. Phys. 18, 025012 (2016).

[9] M. Plihal and A. A. Maradudin, Phys. Rev. B 44, 8565 (1991).

[10] S. Bittner, B. Dietz, M. Miski-Oglu, P. Oria Iriarte, A. Richter, and F. Schäfer, Phys. Rev. B 82, 014301 (2010).

[11] K. Wang, Mater. Res. Express 3, 115007 (2016).

[12] K. Wang, Phys. Rev. B 97, 125146 (2018).

[13] F. D. M. Haldane and S. Raghu, Phys. Rev. Lett. 100, 013904 (2008).

[14] K. Wang, J. Opt. Soc. Am. B 31, 1273 (2014).

[15] R. Saito, G. Dresselhaus, and M. S. Dresselhaus, Phys. Rev. B 61, 2981 (2000).
[16] F. Deng, Y. Sun, X. Wang, R. Xue, Y. Li, H. Jiang, Y. Shi, K. Chang, and H. Chen, Opt. Express 22, 23605 (2014).

[17] Z. Wang and F. Liu, ACS Nano 4, 2459 (2010).

[18] C. Park, J. Appl. Phys. 118, 244301 (2015).

[19] M. M. Sigalas, C. T. Chan, K. M. Ho, and C. M. Soukoulis, Phys. Rev. B 52, 11744 (1995).

[20] K. Wang, Phys. Rev. B 100, 115140 (2019).

[21] See, for example, N. W. Ashcroft and N. D. Mermin, Solid State Physics (Saunders College, Philadelphia, 1976), Chap. 10.

[22] See, for example, J. R. Ferraro and K. Nakamoto, Introductory Raman Spectroscopy (Academic Press, New York, 1994), pp. 310 and 337.

[23] I. N. Levine, Quantum Chemistry, 7th ed. (Pearson, Upper Saddle River, NJ, 2014), pp. 606-609. 